\title{
TOWARD A COSMOLOGICAL HUBBLE DIAGRAM FOR TYPE II-P SUPERNOVAE
}

\author{
Peter Nugent, ${ }^{1}$ Mark Sullivan, ${ }^{2}$ Richard Ellis, ${ }^{3}$ Avishay Gal-Yam, ${ }^{3,4}$ Douglas C. Leonard, ${ }^{3,5}$ D. Andrew Howell, ${ }^{2}$ \\ Pierre Astier, ${ }^{6}$ Raymond G. Carlberg, ${ }^{2}$ Alex Conley ${ }^{2}$ Sebastien Fabbro, ${ }^{7}$ Dominique Fouchez, ${ }^{8}$ \\ James D. Neill, ${ }^{9}$ Reynald Pain, ${ }^{6}$ Kathy Perrett, ${ }^{2}$ Chris J. Pritchet, ${ }^{9}$ and Nicolas Regnault ${ }^{6}$ \\ Received 2005 April 12; accepted 2006 March 20
}

\begin{abstract}
We present the first high-redshift Hubble diagram for Type II-P supernovae (SNe II-P) based on five events at redshift up to $z \sim 0.3$. This diagram was constructed using photometry from the Canada-France-Hawaii Telescope Supernova Legacy Survey and absorption-line spectroscopy from the Keck Observatory. The method used to measure distances to these supernovae is based on recent work by Hamuy \& Pinto and exploits a correlation between the absolute brightness of SNe II-P and the expansion velocities derived from the minimum of the Fe II $\lambda 5169 \mathrm{P}$ Cygni feature observed during the plateau phases. We present three refinements to this method that significantly improve the practicality of measuring the distances of SNe II-P at cosmologically interesting redshifts. These are an extinction correction measurement based on the $V-I$ colors at day 50, a cross-correlation measurement for the expansion velocity, and the ability to extrapolate such velocities accurately over almost the entire plateau phase. We apply this revised method to our data set of high-redshift SNe II-P and find that the resulting Hubble diagram has a scatter of only $0.26 \mathrm{mag}$, thus demonstrating the feasibility of measuring the expansion history, with present facilities, using a method independent of that based on supernovae of Type Ia.
\end{abstract}

Subject headings: distance scale — supernovae: general

Online material: color figures

\section{INTRODUCTION}

The discovery of a cosmic acceleration based on the analysis of the Hubble diagram of Type Ia supernovae (SNe Ia; Riess et al. 1998; Perlmutter et al. 1999) has far-reaching implications for our understanding of the universe. While indirect evidence for the acceleration can be deduced from a combination of studies of the cosmic microwave background and large-scale structure (Efstathiou et al. 2002; Bennett et al. 2003; Eisenstein et al. 2005), distance measurements to $\mathrm{SNe}$ provide a valuable direct and model independent tracer of the evolution of the expansion scale factor necessary to constrain the nature of the proposed dark energy. The mystery of dark energy lies at the crossroads of astronomy and fundamental physics: the former is tasked with measuring its properties and the latter with explaining its origin.

Systematic uncertainties (rather than statistical errors) may soon limit SN Ia measurements of the expansion rate at $z \sim 0.5$ (see Knop et al. 2003; Astier et al. 2005, for recent analyses). A largely unexplored source of potential bias is evolution in the progenitor properties and/or the SN explosion. While several programs are underway to measure, test, and constrain SN Ia system-

\footnotetext{
1 Lawrence Berkeley National Laboratory, 1 Cyclotron Road, Berkeley, CA 94720.

${ }^{2}$ University of Toronto, 60 St. George Street, Toronto, ON M5S 3H8, Canada.

3 California Institute of Technology, 1200 East California Boulevard, Pasadena CA 91125.

${ }^{4}$ Hubble Postdoctoral Fellow.

5 NSF Astronomy and Astrophysics Postdoctoral Fellow.

${ }^{6}$ Laboratoire de Physique Nucleaire et de Haute Energies de Paris (LPNHE), CNRS-IN2P3; and University of Paris VI and VII, 75005 Paris, France.

7 CENTRA, Centro Multidisciplinar de Astrofísica, Instituto Superior Técnico, Avenida Rovisco Pais, 1049 Lisbon, Portugal.

${ }^{8}$ Centre de Physique des Particules de Marseille (CPPM), CNRS-IN2P3 and University Aix Marseille II, Case 907, 13288 Marseille Cedex 9, France.

9 Department of Physics and Astronomy, University of Victoria, P.O. Box 3055, Victoria, BC V8W 3P6, Canada.
}

atics (Sullivan et al. 2005; R. S. Ellis et al. 2006, in preparation), it is highly desirable to consider independent tests of the cosmology in which both the underlying physics and susceptibility to bias and evolution are different.

As cosmological probes, SNe II have lagged behind their brighter and better calibrated cousins, SNe Ia, but their potential has improved significantly as a result of several recent studies. Baron et al. (2003, 2004), Mitchell et al. (2002), and two doctoral theses (Hamuy 2002; Leonard 2000) have used new samples of SNe II and demonstrated that a subset, the plateau SNe II-P, are particularly promising as distance indicators. From an astrophysical standpoint, SNe II-P hold three advantages over SNe Ia as cosmological probes: (1) their progenitor stars are well understood (Heger et al. 2003; Li et al. 2005; Smartt et al. 2003); (2) the physics of their atmospheres, dominated by hydrogen, is much simpler to understand and model (Baron et al. 2004); and (3) while fainter, they are more abundant per unit volume (Mannucci et al. 2005; Cappellaro et al. 2005). The two main disadvantages are that they are on average $1.2 \mathrm{mag}$ fainter in the optical than SNe Ia and that all distance measurements currently based on $\mathrm{SNe}$ II-P require a reasonable quality spectrum of the event.

Unlike the other members of the core-collapse supernova family, SNe II-P maintain a massive hydrogen envelope prior to explosion. From analyses of their optical light curves and spectra (e.g., Chugai 1994), they evidently suffer little subsequent interaction with the surrounding medium - they are the result of the putative red supergiant exploding into a near vacuum. Recent results from spectropolarimetric studies also suggest that, at least during the plateau epoch, the ejecta and electron scattering photosphere are quite spherical (see Leonard \& Filippenko 2005 and references therein).

For SNe II-P, distance measurements from the spectral expanding atmosphere method (SEAM; see Baron et al. 2004), the descendent of the traditional expanding photosphere method (EPM; 
see Kirshner \& Kwan 1974; Schmidt 1993), can be replaced by a more practical empirical method that requires less input data, as developed in Hamuy \& Pinto (2002) and Hamuy (2003) (hereafter HP02 and H03, respectively). The approach advocated by HP02 is a particularly significant development, since it is motivated by sound physical principles. In more luminous supernovae, the hydrogen recombination front is maintained at higher velocities, pushing the photosphere farther out in radius. When a SN II-P is on the plateau phase, a period that lasts for around 100 days, a strong correlation is expected and observed between the velocity of the weak Fe II lines near $5000 \AA$ (which suitably track the electron scattering photosphere) and the luminosity.

Calibration of these standardized candles requires line-ofsight extinction corrections, which can be determined in one of two ways. When the supernova leaves the plateau phase, the photospheric temperature should be close to that of recombination, and thus the observed color at this point will provide a reddening estimate. Alternatively, for well-studied SNe II-P, one can employ detailed modeling of a series of high signal-to-noise ratio $(\mathrm{S} / \mathrm{N})$ spectra to determine the extinction. Here, deviations between the line profiles of selected absorption features and those expected given the observed spectral energy distribution (SED) can be used to estimate selective extinction. Application of these techniques to $24 \mathrm{SNe}$ II-P in the Hubble flow (Fig. 1 of H03) yields an $I$-band Hubble diagram with a scatter of only $0.29 \mathrm{mag}$, corresponding to a precision of $15 \%$ in distance.

The present paper is concerned with extending the HP02 method so that it can be used at cosmological distances. Although economical compared to the SEAM/EPM methods in terms of input data, the HP02 method is still poorly suited as a basis for verifying the cosmic acceleration due to the difficult demands on spectroscopy and the photometric measurements necessary for an extinction correction at high redshift. Our discussion is timely because of the feasibility of locating distant SNe II-P from comprehensive "rolling searches" such as the Canada-France-Hawaii Telescope SN Legacy Survey (SNLS; Astier et al. 2005). Such surveys can generate SNe II-P with $0.1<z<0.4$ continuously with tight constraints on their explosion dates and excellent photometric coverage, making prescheduled spectroscopic campaigns to measure the expansion rate during the plateau phase a practical proposition.

A plan of the paper follows. In $\S 2$ we introduce an enhanced local data set suitable for exploring the potential of an improved method and detail the difficulties we need to overcome to use the HP02 method at intermediate redshifts. We then introduce two extensions to the method and show, via our data set, that we can retain the precision with indicators of velocity and extinction more suited to high-redshift data sets. In $\S 3$ we present the first observations of high-redshift SNe II-P obtained via SNLS and construct a Hubble diagram from these supernovae that demonstrate the feasibility of locating and studying high-redshift $\mathrm{SNe}$ II-P for verifying the cosmic acceleration. We summarize our conclusions in $\S 4$.

\section{IMPROVING THE HP02 METHOD}

The drawbacks of the method introduced by HP02 for studies of cosmologically distant SNe II-P are twofold.

Foremost, the extinction correction cannot be measured using colors determined at the end of the plateau phase, since its precise timing would require continuous monitoring, which is impractical for faint supernovae, and at intermediate redshift, SNe II-P discovered prior to the time they explode will typically be too close to the Sun at this stage in their evolution. Moreover, corrections for extinction based on this method in H03 often pro- duced negative results. Likewise, the $\mathrm{S} / \mathrm{N}$ of plateau spectra will generally not be adequate for detailed spectrum synthesis modeling of various line profiles in order to determine the intrinsic SED. We thus seek a more appropriate way to estimate the lineof-sight extinction.

Second, it is a challenge at moderate redshift to secure accurate measures of the weak diagnostic Fe II $\lambda 5169$ line used to measure the photospheric expansion during the plateau phase, even with the largest ground-based telescopes. In particular, as diagnostic lines in this region of the spectrum are redshifted into the $\mathrm{OH}$ forest beyond $z \simeq 0.5$, it becomes advantageous to explore alternatives should the Fe II $\lambda 5169$ line profile be polluted by a sky feature. We therefore wish to explore the practicality of alternative, stronger lines to measure the photospheric expansion.

To explore the possibilities, we have constructed a sample of nearby SNe II-P that includes all of those in H03 for which there is both $V$ - and $I$-band data. We also include SN 2004dh, a SN discovered by the Lick Observatory Supernovae Search (Moore $\&$ Li 2004), and followed as part of the Caltech Core-Collapse Program (CCCP; see Gal-Yam et al. 2004, 2006a [in preparation]), which leads to a total of 19 SNe II-P. We have updated the distances to two supernovae presented in H03. In the case of SN 1999em we use the Hubble Space Telescope (HST)-determined Cepheid distance to the host (Leonard et al. 2003). For SN 1999gi we use the improved distance derived via a variety of methods as discussed in Leonard et al. (2002). These changes have very little effect on the overall fit, as both galaxies are nearby with large uncertainties in their relative distances. We have also removed all of the extinction corrections applied in H03.

We now turn to two improvements necessary to enable us to use $\mathrm{SNe}$ II-P as distance indicators at moderate redshift.

\subsection{Incorporating $V-I$ Colors}

The first modification of the HP02 method we have explored makes use of the rest-frame $V-I$ color during the plateau phase at day 50 to perform an extinction correction rather than relying on colors at the end of the plateau phase or on detailed modeling, as in H02. Our goal is to explore empirically the extent to which we can retain a tight $\mathrm{Fe}$ II velocity-luminosity relation using the observed $V-I$ color at this epoch.

If we fit for the velocity and luminosity using plateau-phase data, interpolated to day 50 , we find

$M_{I}=-\alpha \log _{10}\left(V_{\mathrm{Fe}} / 5000\right)-1.36\left[(V-I)-(V-I)_{0}\right]+M_{I_{0}}$.

Here, as in $\mathrm{H} 03$, we have adopted the relative surface brightness fluctuation distance scale (Tonry et al. 2000). The resulting fit to the data yields $\alpha=6.69 \pm 0.50$ and $M_{I_{0}}=-17.49 \pm$ $0.08\left(H_{0}=70 \mathrm{~km} \mathrm{~s}^{-1} \mathrm{Mpc}^{-1}\right)$ for a $(V-I)_{0}=0.53$. In this fit we have employed the standard relationship between the $V-I$ colors for a dust law with $R_{V}=3.1$ for a SN II-P at day $50\left(A_{I}=\right.$ $\left.1.36 E_{V-I}\right)$. As is done in the SN Ia studies (Guy et al. 2005) for the color-stretch relationship, we have adopted a ridgeline, unextinguished $(V-I)_{0}$ color for SN II-P of $0.53 \mathrm{mag}$. Under the assumption that the extinction laws are similar from galaxy to galaxy, the exact choice is irrelevant, since this term and the $M_{I_{0}}$ term are degenerate for the purpose of using these objects as cosmological probes. Using this technique we can produce a Hubble diagram in rest-frame $I$ band with a scatter of only 0.28 mag (Fig. 1) for those SNe II-P in the Hubble flow ( $c z>$ $3000 \mathrm{~km} \mathrm{~s}^{-1}$ ), similar to that found in H03. The scatter for all SN II-P is reduced from 1.11 to 0.52 mag. Crucially, we find 


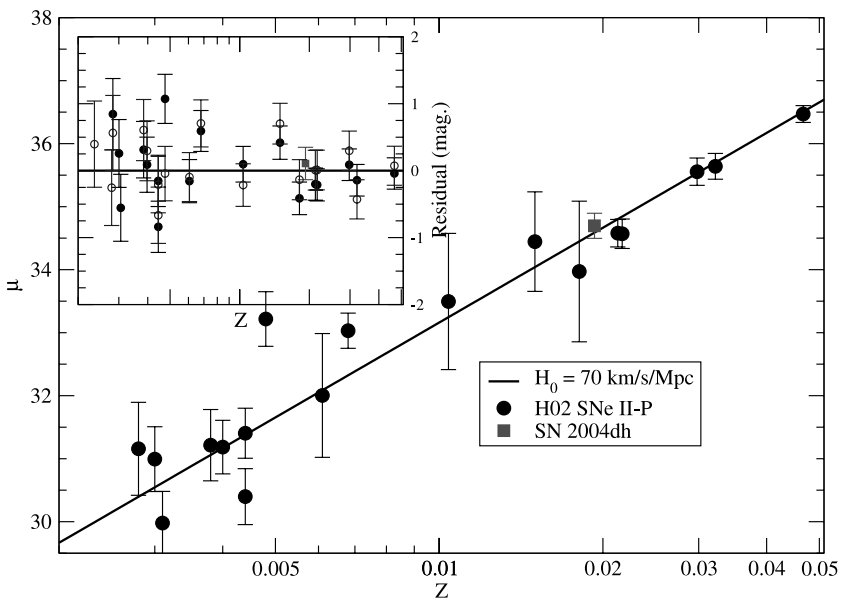

FIG. 1.-Revised Hubble diagram (scaled to $H_{0}=70 \mathrm{~km} \mathrm{~s}^{-1} \mathrm{Mpc}^{-1}$ ) for local SNe II-P (circles and square) using an improved estimator for extinction derived from $V-I$ colors and $\mathrm{Fe}$ II velocities observed during the plateau phase at day 50. Inset: Residual Hubble diagram for both the revised method ( filled circles) and the data under the assumption that they are pure standard candles in $I$ band, corrected only for MW extinction (open circles). The scatter for those SN II-P in the Hubble flow is reduced from 0.59 to $0.28 \mathrm{mag}$, similar to the 0.29 mag originally achieved in $\mathrm{H} 03$ and based on observables substantially better suited to high-redshift observations. The scatter for all SN II-P is reduced from 1.11 to $0.52 \mathrm{mag}$. [See the electronic edition of the Journal for a color version of this figure.]

there is no advantage in using reddening estimators based on latetime color measures or on detailed modeling of the spectroscopic data.

To address the robustness of this simple approach, we investigated solutions in which we permit an additional parameter based on a possible relationship between the $\mathrm{Fe}$ II velocity and the dereddened $V-I$ color. One could easily envision a correlation that brighter SN II-P were faster and bluer. However, the correlation we find is weak and lacks any statistical significance.

We note that for $\alpha \neq 5.0$ ( $\alpha=5$ implies that the luminosity follows the square of the radius only), there presumably does exist an additional correlation between the effective temperature and density of the expanding photosphere and its radius, so conceivably there is some scope for further improvement in defining equation (1). Future work, such as the ongoing research by the CCCP and the Carnegie Supernova Project (CSP; Freedman 2005), will be helpful in clarifying the possibilities and allow us to address the strongly correlated issues of extinction corrections and velocity-dependent colors.

\subsection{Using Alternative Lines to Diagnose the Photospheric Expansion}

We now explore whether alternative absorption lines can be used in addition to $\mathrm{Fe}$ II $\lambda 5169$ as diagnostics of the photospheric expansion velocity. Over the wavelength range 4500-5500 $\AA$ there exist several Fe II lines (the strongest of which are at 4924, 5018, and $5169 \AA$ ). Moreover, often the $\mathrm{H} \beta \lambda 4861$ absorption line is prominent. Depending on the supernova and its phase, the relative strengths of these lines can vary considerably (see Fig. 2). In general, at earlier epochs $\mathrm{H} \beta$ tends to dominate, while at later epochs the Fe II lines are the dominant absorption features over this wavelength range. Accordingly, if at higher redshift we are able to compare to any or all of these lines, we can minimize the effects of the interference with $\mathrm{OH}$ night-sky features and any underlying host galaxy contamination.

In order to explore possible systematic effects arising from using different absorption lines as measures of the expansion, we

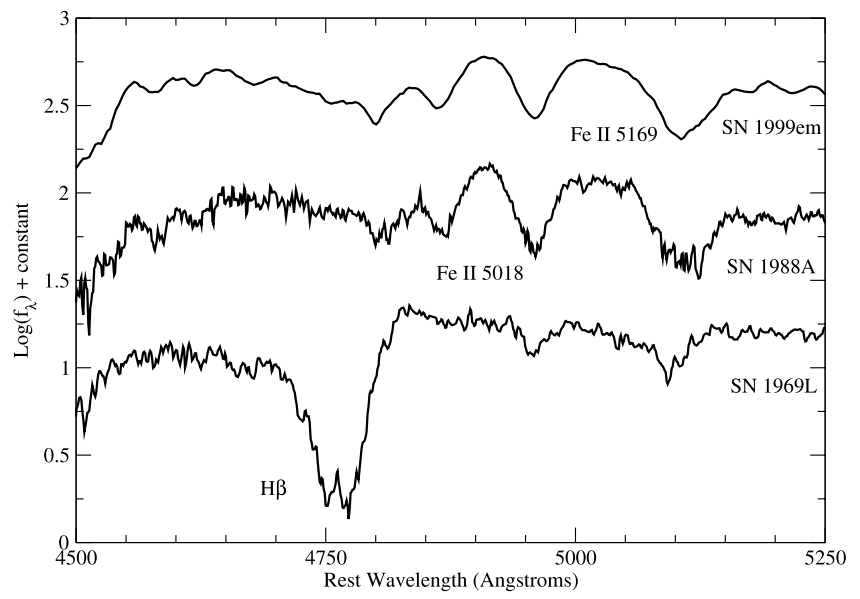

FIG. 2.-Comparison of two Fe II-dominated spectra to an $\mathrm{H} \beta$-dominated spectrum. This demarcation is defined by the equivalent width of $\mathrm{H} \beta$ being greater than the sum of the Fe II features. Depending on the SN and its phase, any one of these features could potentially be the strongest and/or the most easily measured (due to sky lines or host galaxy contamination) and thus could be used at high redshift.

first examine trends in local data. To do this we assembled a library of SNe II-P spectra based on data from the SUSPECT database ${ }^{10}$ for the following: SNe 1969L, 1988A, 1988H, 1993W, 1999gi, and 1999em (Benetti 1991; Turatto et al. 1993; Leonard et al. 2002, 2003; Hamuy et al. 2001; Baron et al. 2003) as well as data from the first year of the CCCP: SNe 2004A, 2004T, 2004dh, 2004du, 2004em, and 2004et (A. Gal-Yam et al. 2006b, in preparation). A particularly important question is whether it is safe to use $\mathrm{H} \beta$ as a diagnostic rather than the $\mathrm{Fe}$ II lines, given the former is often quite strong. In what follows, we have divided the local data set into two categories: those dominated by the $\mathrm{H} \beta$ feature and those dominated by the $\mathrm{Fe}$ II features (defined according to whether the equivalent width of $\mathrm{H} \beta$ is respectively greater than or less than that of the sum of the $\mathrm{Fe}$ II features). The motivation here

${ }^{10}$ See http://bruford.nhn.ou.edu/ suspect/index $1 . \mathrm{html}$.

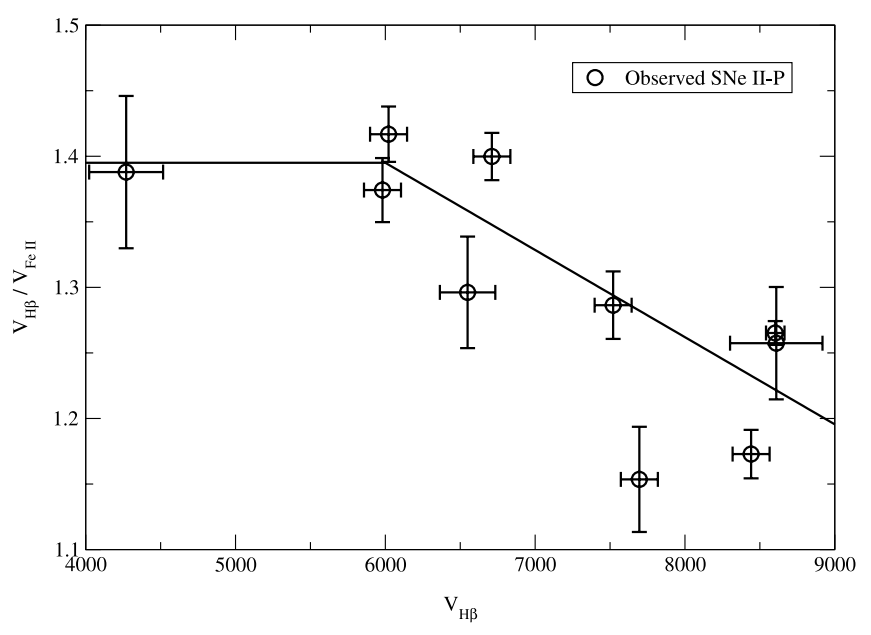

FIg. 3.- Ratio of the velocities of $\mathrm{H} \beta$ to $\mathrm{Fe}$ II $\lambda 5169$ vs. the $\mathrm{H} \beta$ velocity in the $\mathrm{H} \beta$-dominated spectra. We fit this ratio in two pieces: a constant for velocities below $6000 \mathrm{~km} \mathrm{~s}^{-1}($ ratio $=1.395)$ and linearly for higher velocities (ratio = $\left.(1.395-6.489) \times 10^{-5}[V(\mathrm{H} \beta)-6000]\right)$. The linear portion is the only one relevant to our analysis, as our high-redshift data lie within these velocities. The dispersion about this relationship $( \pm 0.054)$ translates to an uncertainty in measuring the $\mathrm{Fe}$ II velocity from $\mathrm{H} \beta$ of $\sim 300 \mathrm{~km} \mathrm{~s}^{-1}$. See text for a full discussion and explanation of this trend. 


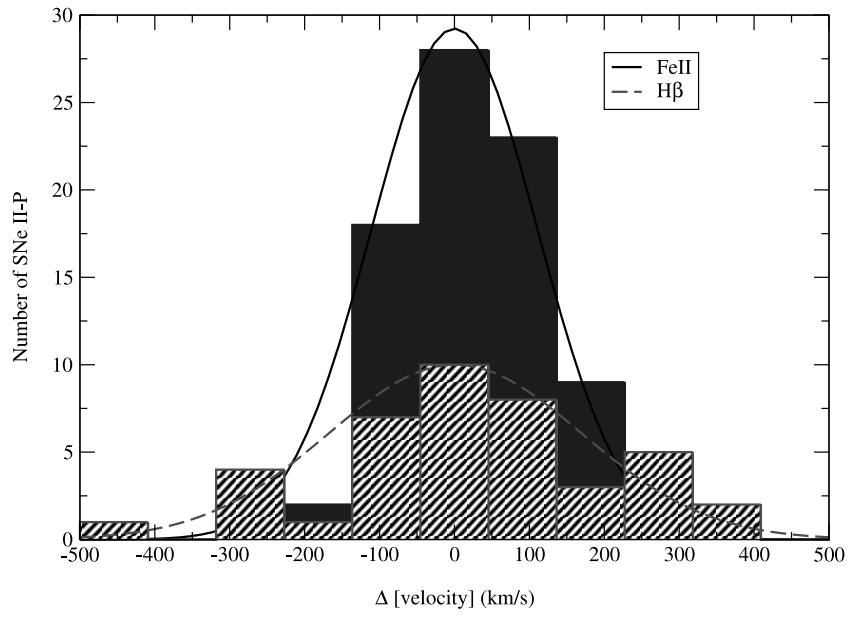

FIG. 4.- - Histogram of the difference in measured velocity vs. the velocity derived from the cross-correlation technique for $\mathrm{Fe}$ II $\lambda 5169$ and $\mathrm{H} \beta$. Here we have compared each SN II-P in our library to all the others, splitting the comparison library for SN II-P to those that are dominated by $\mathrm{H} \beta$ and those by Fe II. The measured dispersion is $161 \mathrm{~km} \mathrm{~s}^{-1}$ for the $\mathrm{H} \beta$ subset and $108 \mathrm{~km} \mathrm{~s}^{-1}$ for the Fe II subset. [See the electronic edition of the Journal for a color version of this figure.]

is twofold. First, unlike $\mathrm{H} \beta$, in the case of the Fe II-dominated spectra, the weak lines form at the same location in the atmosphere near the electron scattering photosphere. Thus we might expect any (or all) of them to be acceptable for measuring the velocity. Second, as we later test a novel cross-correlation method across this wavelength range, it is very helpful to understand possible systematic velocity differences between $\mathrm{H} \beta$ and the $\mathrm{Fe}$ II lines. For both $\mathrm{Fe}$ II $\lambda 5169$ and $\mathrm{H} \beta$, we measured absorption velocities using

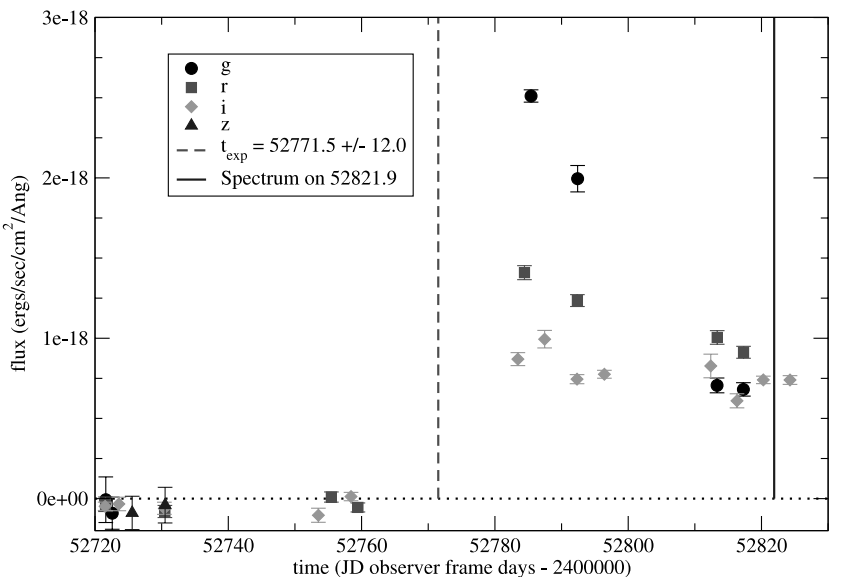

FIG. 6. - SNLS $g^{\prime} r^{\prime} i^{\prime} z^{\prime}$ light curves (in $f_{2}$ ) for SNLS-03D3ce at $z=0.2881$. Unfortunately, this SN was not observed in $z^{\prime}$ on the plateau. At this redshift $z^{\prime}$ overlaps nicely with the rest-frame $I$ band; thus, the $V-I$ color had to be extrapolated from bluer colors than desired for this SN II-P. [See the electronic edition of the Journal for a color version of this figure.]

a routine in which we first subtract the continuum, take the wavelength derivative and then fitted for the wavelength where this derivative changes sign.

Figure 3 shows that, in the $\mathrm{H} \beta$-dominated spectra, the velocity of $\mathrm{H} \beta$ is significantly higher than that of $\mathrm{Fe}$ II $\lambda 5169$ with the trend that the ratio of $\mathrm{H} \beta$ to $\mathrm{Fe}$ II drops toward unity only at very high velocities. Clearly, simply replacing Fe II absorption velocities with measures of $\mathrm{H} \beta$ would lead to erroneous results. However, if we fit this ratio in two sections, a constant for velocities below $6000 \mathrm{~km} \mathrm{~s}^{-1}($ ratio $=1.395)$ and a linear decline for higher velocities $\left(\right.$ ratio $\left.=(1.395-6.489) \times 10^{-5}[V(\mathrm{H} \beta)-6000]\right)$, the
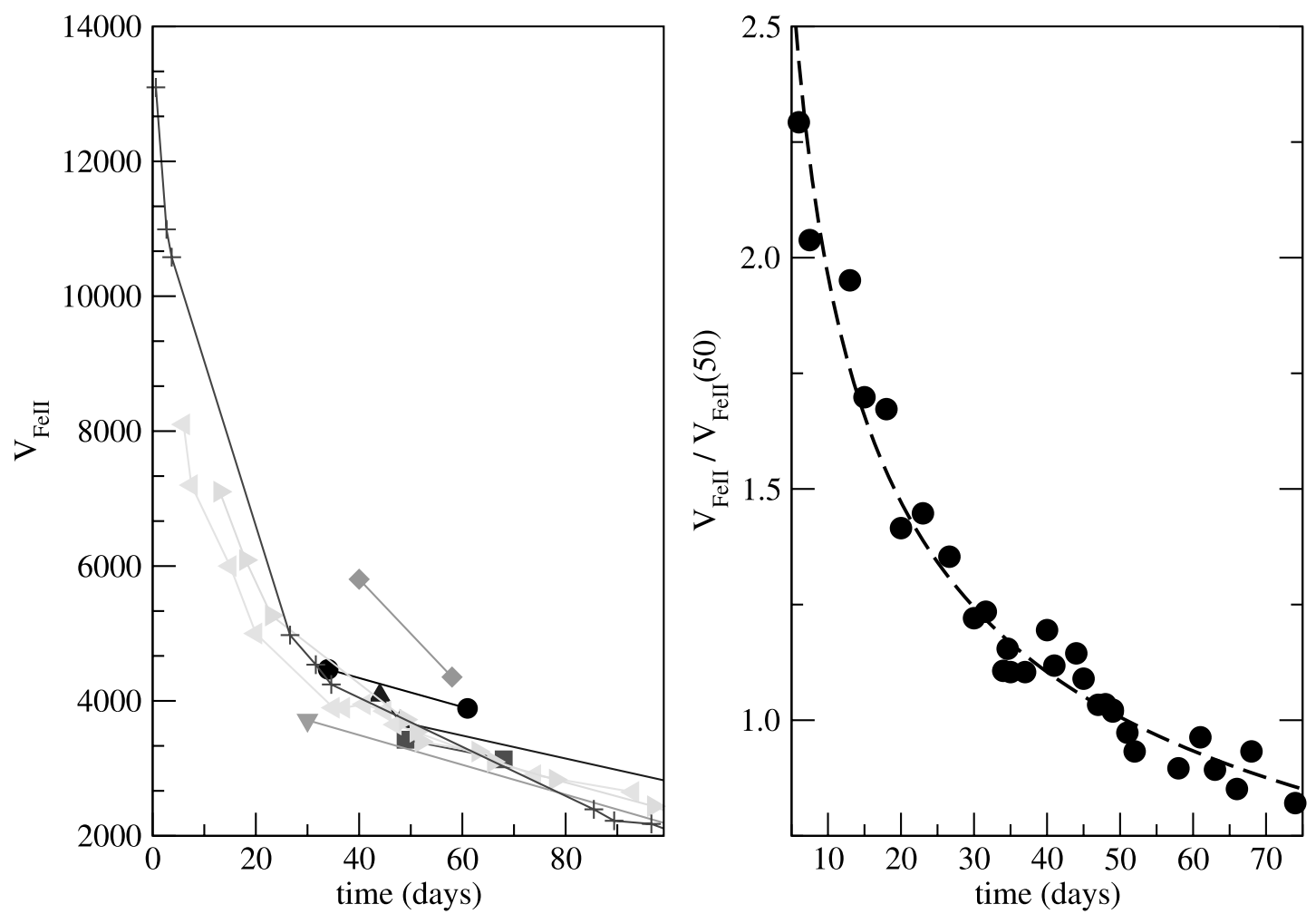

FIG. 5. - Left: Plot of the velocity of Fe II $\lambda 5169$ vs. time for the published SNe II-P (each symbol represents an individual SN). Right: Plot of the individual SNe II-P with their velocities normalized to day 50, along with the best fit for the evolution of the decline covering the epochs for our high-redshift SNe (day 9-75). The fit is a power law of the form $V(50)=V(t)(t / 50)^{0.464 \pm 0.017}$. [See the electronic edition of the Journal for a color version of this figure.] 


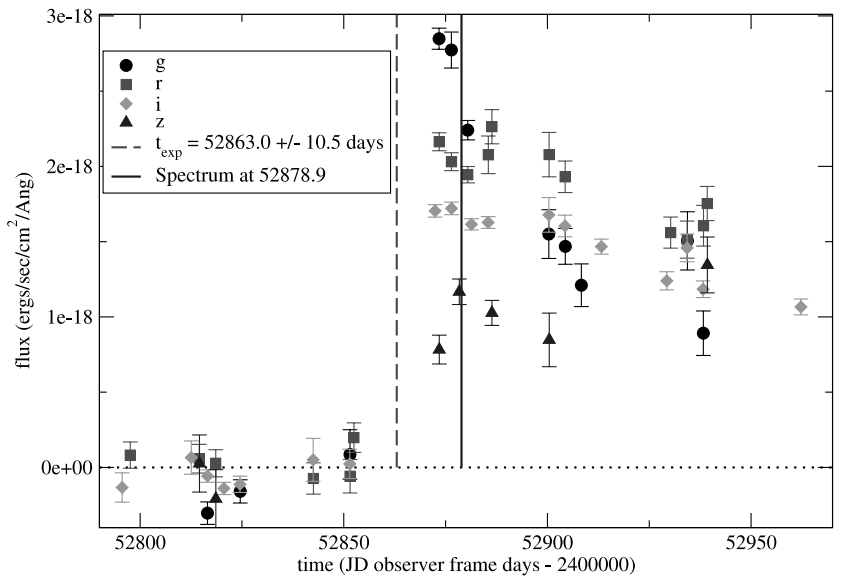

FIG. 7.-SNLS $g^{\prime} r^{\prime} i^{\prime} z^{\prime}$ light curves (in $f_{\lambda}$ ) for SNLS-03D4cw at $z=0.1543$. [See the electronic edition of the Journal for a color version of this figure.]

dispersion about this relationship $( \pm 0.054)$ translates to an uncertainty in estimating the $\mathrm{Fe}$ II velocity from $\mathrm{H} \beta$ measures of only $\sim 300 \mathrm{~km} \mathrm{~s}^{-1}$.

Of course, before accepting such an empirical correction, it is important to understand the trend physically. To accomplish this, we examined spectrum synthesis fits to model SNe II-P discussed by Baron et al. (2004). The predicted trend is qualitatively similar in form to that observed and, in particular, reproduces the decline in the ratio observed at higher velocities. The model spectra with higher effective temperatures were in general from earlier epochs with higher velocities, while the cooler ones were later with lower velocities. The optical depth of $\mathrm{H} \beta$ is strongly tied to the level of ionization of hydrogen. Thus as the temperature drops, the velocities fall, less hydrogen is ionized, and the optical depth of $\mathrm{H} \beta$ increases significantly compared to the weak Fe II lines. This in turn increases the ratio.

In order to maximize the information content in either the series of weak $\mathrm{Fe}$ II lines or in the $\mathrm{H} \beta$-dominated spectra, we then examined a cross-correlation analysis across the wavelength range 4500-5500 $\AA$. In the Fe II-dominated spectra we simply compared the measured $\mathrm{Fe}$ II $\lambda 5169$ velocities to those obtained in the cross-correlation analysis. For the $\mathrm{H} \beta$-dominated spectra we compare the measured $\mathrm{H} \beta$ velocities and then reduced these to equivalent $\mathrm{Fe}$ II $\lambda 5169$ velocities using the fit to the ratio defined above. In performing cross-correlations, all spectra are continuum subtracted and analyzed in log wavelength space in the manner described by Tonry \& Davis (1979).

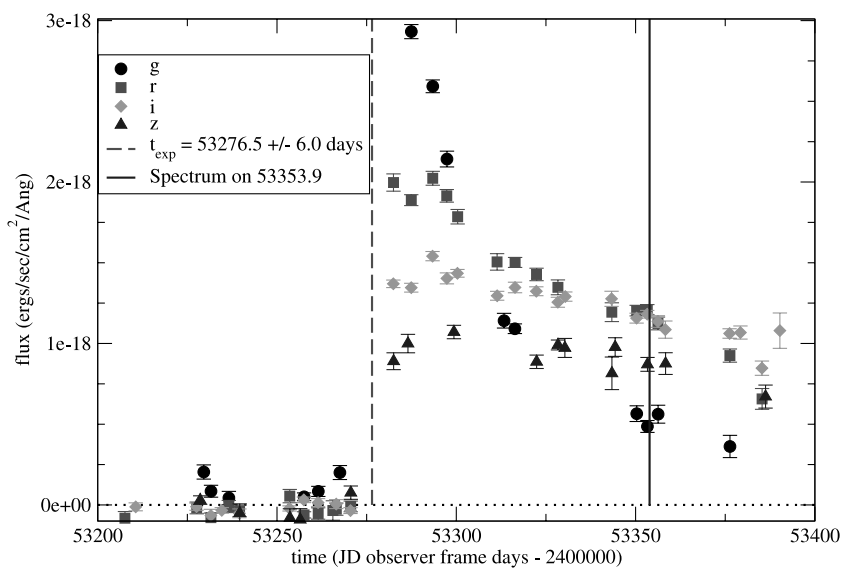

FIG. 8. - SNLS $g^{\prime} r^{\prime} i^{\prime} z^{\prime}$ light curves (in $f_{\lambda}$ ) for SNLS-04D1ln at $z=0.2078$. [See the electronic edition of the Journal for a color version of this figure.]

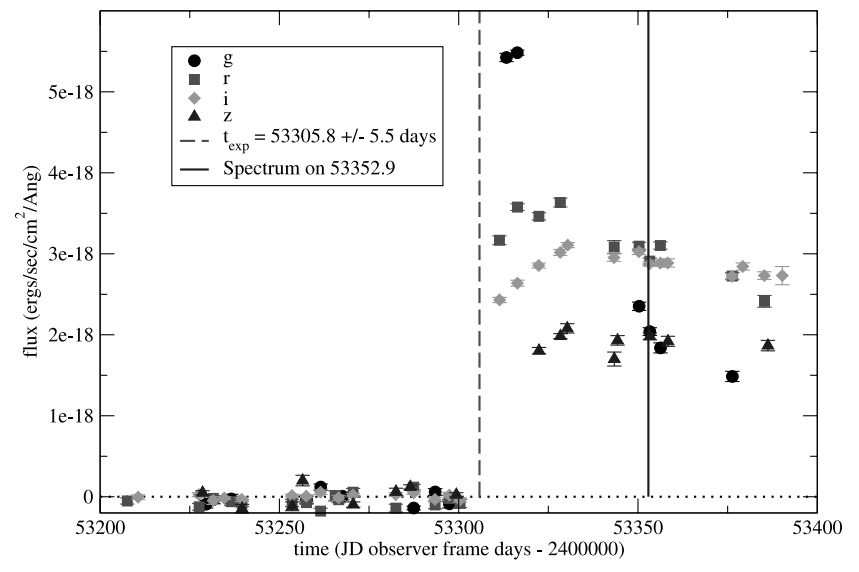

FIG. 9.-SNLS $g^{\prime} r^{\prime} i^{\prime} z^{\prime}$ light curves (in $f_{\lambda}$ ) for SNLS-04D1pj at $z=0.1556$. [See the electronic edition of the Journal for a color version of this figure.]

A measure of the potential bias and systematic uncertainty arising in the cross-correlation method can be obtained by considering the distribution of velocity differences obtained by multiple template comparisons within our local data set (Fig. 4). Each local supernova, when compared to the average of the crosscorrelation calculated velocities, shows differences of typically $<150 \mathrm{~km} \mathrm{~s}^{-1}$. This is a measure of template mismatch for the local sample in the wavelength region sample: i.e., in individual SNe II-P, the strongest features present can form at slightly different velocities than $\mathrm{Fe}$ II $\lambda 25169$, in addition to measurement uncertainties for the minimum of that line in our spectral library. This bias is recorded for each of our template spectra and subtracted off when used in the cross-correlation measurement. The resulting measured dispersion in comparing one of our template spectra to the rest in their respective sets is $160 \mathrm{~km} \mathrm{~s}^{-1}$ for $\mathrm{H} \beta$-dominated spectra and $110 \mathrm{~km} \mathrm{~s}^{-1}$ for the $\mathrm{Fe}$ II-dominated spectra.

Of course, these tests calibrate systematic uncertainties that are dominant only for low-redshift SNe IIP. At high redshift we can expect an additional component from the lower $\mathrm{S} / \mathrm{N}$ implicit in the fainter sources. To estimate how effective this method will be at intermediate redshift, where our $\mathrm{S} / \mathrm{N}$ is typically between 8 and 20 per $2 \AA$ over the relevant wavelength range, we performed a Monte Carlo equivalent of the above test by degrading the $\mathrm{S} / \mathrm{N}$ of the nearby spectra to 7 per $2 \AA$. The resulting dispersion for individual measurements increased by only $15 \mathrm{~km} \mathrm{~s}^{-1}$,

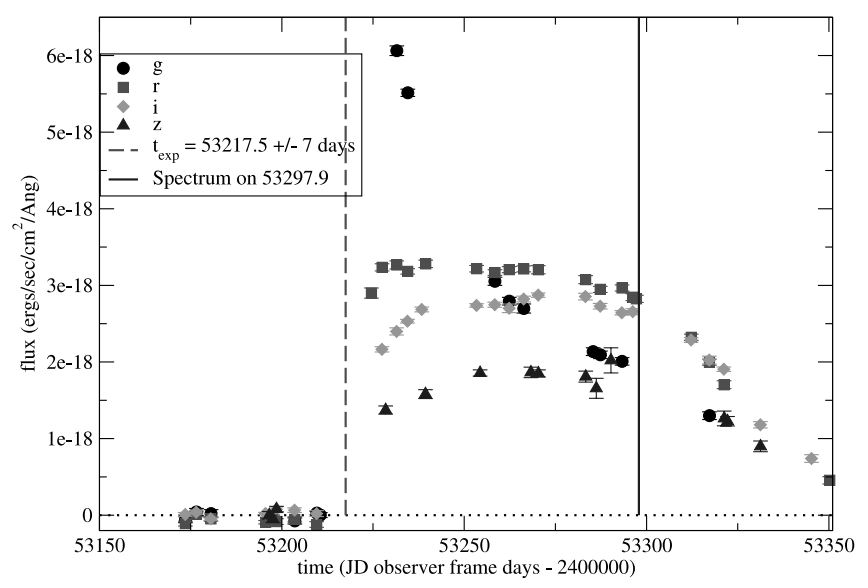

FIG. 10.-SNLS $g^{\prime} r^{\prime} i^{\prime} z^{\prime}$ light curves (in $f_{\lambda}$ ) for SNLS-04D4fu at $z=0.1330$. This SN was caught just after shock breakout as the $g^{\prime} r^{\prime} i^{\prime} z^{\prime}$ colors (uncorrected for extinction) lead to a temperature $>20,000 \mathrm{~K}$. [See the electronic edition of the Journal for a color version of this figure.] 


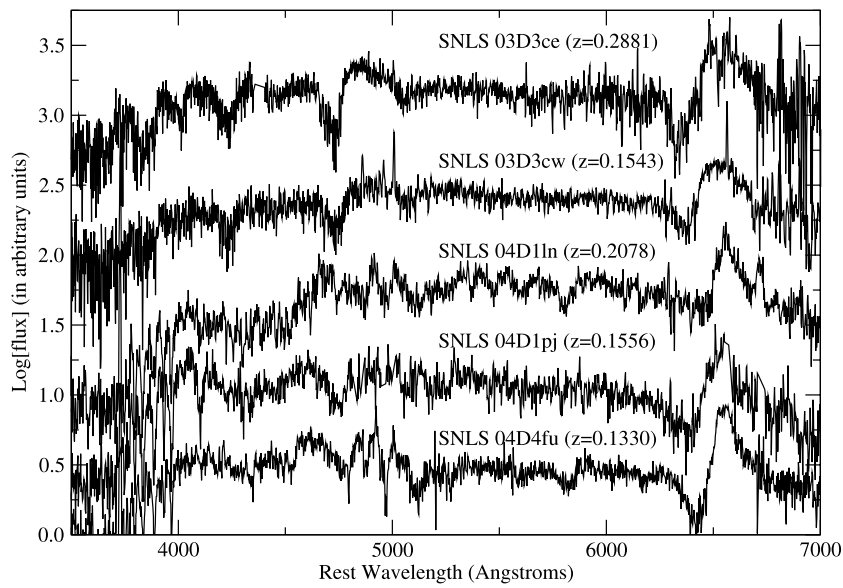

FIG. 11.- Rest-frame spectra of the high-redshift SNe II-P observed at Keck+LRIS in 2003 and 2004. Top to bottom: SNLS 03D3ce, 03D4cw, 04D1ln, $04 \mathrm{D} 1 \mathrm{pj}$, and 04D4fu. The quality of these spectra is such that the measurement of the $\mathrm{Fe}$ II velocities, based on the technique described in $\S 2.2$ over the range $4500-5500 \AA$, is good to $\sim 250 \mathrm{~km} \mathrm{~s}^{-1}$.

clearly showing that the cross-correlation method is sufficiently accurate for our purpose.

The final problem we must overcome in facilitating a cosmological application of SNe II-P concerns the fact that it is impractical to schedule, in advance, a spectroscopic night at day 50 for each supernova of interest. Thus we need to explore how well we can extrapolate the velocity from a given epoch to day 50 . While published data with the run of velocity with time are limited, that available to us indicates that the time dependence of the Fe II $\lambda 5169$ velocity can be well represented by a power law of the form

$$
V(50)=V(t)(t / 50)^{0.464 \pm 0.017}
$$

(see Fig. 5). Extrapolation for epochs between 9 and 75 days to day 50 can be made quite reliably adding an uncertainty of $<175 \mathrm{~km} \mathrm{~s}^{-1}$. Adding the various uncertainties in quadrature indicates expansion velocities of distant SNe II-P should be typically accurate to $\simeq 220 \mathrm{~km} \mathrm{~s}^{-1}$.

\section{A COSMOLOGICAL HUBBLE DIAGRAM BASED ON A SAMPLE OF HIGH-REDSHIFT SNe II-P}

In the previous section, we have shown that extinction corrections using $V-I$ colors during the plateau phase at day 50 and concurrent expansion velocities determined using a variety of prominent absorption lines can be used to generalize and extend the important distance determination method initially proposed by HP02. As SNe II-P are now being found to redshifts $z \simeq 0.4$ in rolling searches such as the SNLS (Sullivan et al. 2005), we can now apply our method to these distant supernovae to independently verify the cosmic acceleration.

Starting in 2003, we began using the Low Resolution Imaging Spectrometer (LRIS; Oke et al. 1995), a double-arm spectrograph mounted on the $10 \mathrm{~m}$ Keck I telescope, to observe SNLS-discovered supernovae. Although our primary program initially targeted SNe Ia (R. S. Ellis et al. 2006, in preparation), during the first 2 years we also successfully studied five moderateredshift $(0.1<z<0.3)$ SNe II-P, identified from the SNLS rolling search via their light curves. These events were typically observed 5 times per lunation in $g^{\prime} r^{\prime} i^{\prime} z^{\prime}$ (Astier et al. 2005) with

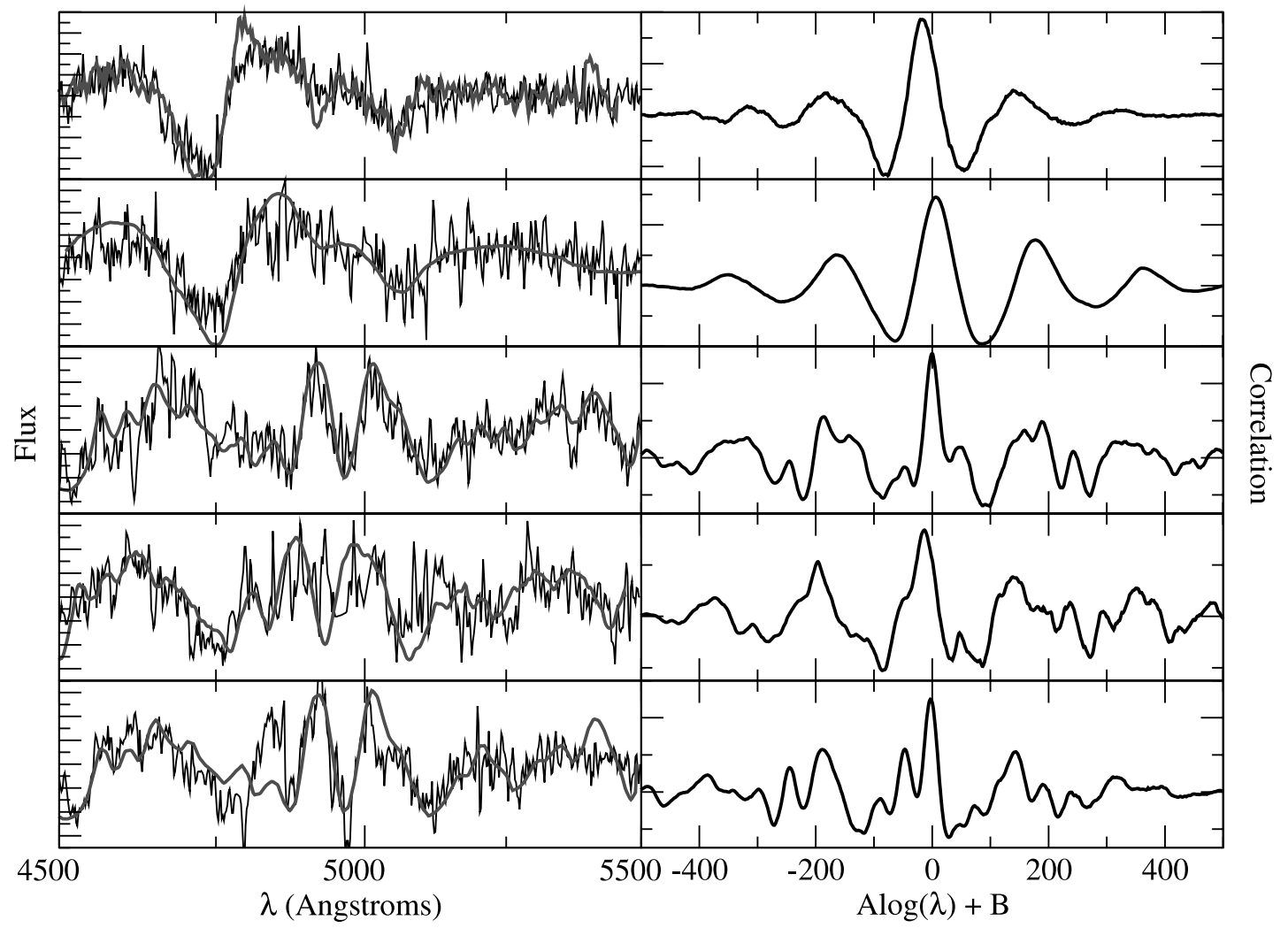

FIG. 12.-Left: Plot of the best-fit template spectrum (thick lines) and the SNLS SNe II-P (thin lines) used in the cross-correlation technique. Right: Resulting correlation. Top to bottom: SNLS-03D3ce, 03D4cw, 04D1ln, 04D1pj, and 04D4fu. [See the electronic edition of the Journal for a color version of this figure.] 
TABLE 1

Cross-Correlation Velocity Analysis for H $\beta$-dominated Spectra

\begin{tabular}{|c|c|c|c|c|}
\hline SNLS Name & Template Spectrum (Epoch) & $\begin{array}{c}\mathrm{H} \beta \text { Velocity } \\
\left(\mathrm{km} \mathrm{s}^{-1}\right)\end{array}$ & $\mathrm{H} \beta / \mathrm{Fe}$ II Ratio & $\begin{array}{c}\text { Fe II Velocity } \\
\left(\mathrm{km} \mathrm{s}^{-1}\right)\end{array}$ \\
\hline 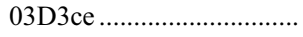 & SN 1999em (15) & $8137.6 \pm 42.0$ & $1.256 \pm 0.054$ & $6479.0 \pm 350$ \\
\hline 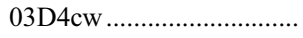 & SN 2004T (15) & $7355.4 \pm 56.3$ & $1.307 \pm 0.054$ & $5627.7 \pm 304$ \\
\hline
\end{tabular}

Notes.- The best match nearby template spectrum from our library along with the resulting median absolute deviation velocity measurement, with a 3 median-absolute-deviations cut, for the $\mathrm{H} \beta$-dominated SNLS supernovae. Also listed are the $\mathrm{H} \beta$ to Fe II ratios and the resulting Fe II velocities. Approximate epochs for the nearby SNe II-P are in parentheses.

coverage starting before explosion and extending throughout the plateau phase (see Figs. 6-10).

We measure $g^{\prime} r^{\prime} i^{\prime} z^{\prime}$ fluxes of the SNLS supernovae using a PSF fitting technique. The first step is to perform a photometric alignment of each SN observation to secondary standards within each field using a multiplicative scaling factor and assign an astrometric solution using custom-built astrometric reference catalogs. Deep "references" are constructed to contain no SN light and are resampled to the pixel coordinate system of each observation. The references are then PSF matched and subtracted from each individual observation, leaving a difference image containing only SN photons. The fluxes are then estimated by fitting a PSF profile measured from stars in the unsubtracted image at the known position of the $\mathrm{SN}$, with the weighting in each pixel equal to $1 / \sigma^{2}$, where $\sigma$ is calculated from the background sky noise and the Poisson noise from the $\mathrm{SN}$ and host photons. An average flux measurement on each night of observation is made using a sigmaclipped weighted average of the individual data frames.

Keck spectra were taken on the plateau over a range in time of $15<t<75$ rest-frame days (see Fig. 11). The Keck-LRIS observing configuration used the 560 dichroic, with the $600 \mathrm{l} / \mathrm{mm}$ grism blazed at $4000 \AA$ on the blue side, and the $4001 / \mathrm{mm}$ grating blazed at $8500 \AA$ on the red side. The grating central wavelength was adjusted to ensure a complete wavelength coverage from 3500 to $9200 \AA$. Depending on seeing conditions, either a $1^{\prime \prime}$ or 0.7 slit was used, and the supernova was dithered between each $1800 \mathrm{~s}$ exposure by $3^{\prime \prime}-5^{\prime \prime}$. Typical total exposure times were $2-3 \mathrm{hr}$, and all data were taken at the parallactic angle. All spectroscopic data were processed using a pipeline developed by one of us (M. S.), which performs basic bias subtraction and flat fielding, as well as a fringe subtraction on the red side using a fringe frame constructed from an entire night's data. Each exposure was extracted separately on the red and blue sides, wavelength and flux calibrated, corrected for atmospheric extinction, and optimally combined with the other exposures to form one continuous spectrum.

To calculate the rest-frame $V-I$ colors during the plateau phase, we first made an estimate of the explosion date of the supernova conservatively based on the midpoint between the last nondetection and the first detection of the SN in any filter during the rolling search. We then interpolated the $g^{\prime} r^{\prime} i^{\prime} z^{\prime}$ colors at day $50(1+z)$. These colors were used to "warp" a day 50 template SN II-P spectrum, redshifted appropriately, following the protocol described in Nugent et al. (2002), with the exception that here we use spline fits to the underlying colors to adjust the template rather than a reddening law. This spectrum was then deredshifted, and the restframe $V-I$ color of the $\mathrm{SN}$ was calculated.

The Fe II velocities were measured via the cross-correlation technique introduced in $\S 2.2$, extrapolated to rest-frame day 50 using equation (2). In determining the velocity and its uncertainty from the full range of local templates, we adopted the median absolute deviation method, which provides a particularly robust measure of the dispersion when there are a few significant outliers. The distribution of cross-correlation velocities is calculated using all local templates and the median of the distribution estimated. The absolute modulus of the deviation from the median velocity is calculated, and that distribution is medianed and $3 \sigma$ clipped. We then take the mean of the remaining velocities and the rms about this mean as our velocity and uncertainty. Typically, this procedure reduced the comparison library by $<25 \%$. The correlations and resulting best fits can be seen in Figure 12 and are listed in Tables 1 and 2.

Appropriately dealing with uncertainties in the above measurements and evaluating their implications on the inferred distances is an important aspect of our analysis. An uncertainty in the explosion date will propagate to one in the distance determination. On the one hand, the expansion velocity will decline (implying a lower luminosity) as the explosion date is moved forward, whereas the SN will get redder with time on the plateau, implying more extinction (and a more luminous event). To quantify such biases, we devised a Monte Carlo simulation incorporating these uncertainties. The results in Table 3 summarize both the measurements and their uncertainties as a result of these simulations. To illustrate the potential value of our Hubble diagram, we performed a cosmological fit using both these and the low-redshift supernova (Table 4) under the assumption of the flat cosmological model found in Astier et al. (2005).

The values of the fitting parameters in this joint fit of both the high- and low-redshift SNe II-P changed to $\alpha=5.81$ and $M_{I_{0}}=$ 17.52. This value of $\alpha$ is lower by $1.5 \sigma$, compared to the fit for only the low-redshift SNe II-P; see Figure 14. The observed scatter was $0.26 \mathrm{mag}$ for all supernovae in the Hubble flow. The Hubble diagram is presented in Figure 13. The reduced $\chi^{2}$ is 1.8 , which implies an intrinsic dispersion of $0.12 \mathrm{mag}$, although this number is quite tentative due to the low number statistics involved in this analysis.

Two of the problems facing this data set of SNe II-P are the data analysis inconsistencies between the low- and high-redshift data and small number statistics. The low-redshift data assembled in $\mathrm{H} 03$ measured and then extrapolated/interpolated the

TABLE 2

Cross-Correlation Velocity Analysis for Fe il-dominated Spectra

\begin{tabular}{|c|c|c|}
\hline SNLS Name & Template Spectrum (Epoch) & $\begin{array}{c}\mathrm{Fe} \text { II Velocity } \\
\quad\left(\mathrm{km} \mathrm{s}^{-1}\right)\end{array}$ \\
\hline 04D1ln .................... & SN 1999em (61) & $3201.8 \pm 86.9$ \\
\hline 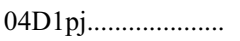 & SN 2004em (35) & $5478.5 \pm 72.5$ \\
\hline 04D4fu ..................... & SN 2004A (50) & $3280.5 \pm 35.1$ \\
\hline
\end{tabular}

NoTES.-The best match nearby template spectrum from our library along with the resulting median absolute deviation velocity measurement, with a 3 medianabsolute-deviations cut, for the Fe II-dominated SNLS supernovae. Approximate epochs for the nearby SNe II-P are in parentheses. 
TABLE 3

Measurements for the SNLS High-Redshift SNe II-P

\begin{tabular}{|c|c|c|c|c|c|c|c|}
\hline SNLS Name & $z$ & $t_{\text {expl }}$ & $t_{\text {spec }}$ & $\begin{array}{c}V_{\mathrm{Fe} \mathrm{II}} \\
\left(\mathrm{km} \mathrm{s}^{-1}\right)\end{array}$ & $I$ & $V-I$ & $\mu$ \\
\hline 03D3ce.. & 0.2881 & $2771.5 \pm 12.0$ & 2821.9 & $5762 \pm 522$ & $23.45 \pm 0.50$ & $0.39 \pm 0.50$ & $41.10 \pm 0.40$ \\
\hline 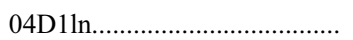 & 0.2077 & $3276.5 \pm 6.0$ & 3353.9 & $3593 \pm 159$ & $22.79 \pm 0.05$ & $0.70 \pm 0.07$ & $39.70 \pm 0.15$ \\
\hline 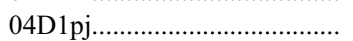 & 0.1556 & $3305.8 \pm 5.5$ & 3352.9 & $4981 \pm 214$ & $21.99 \pm 0.04$ & $0.66 \pm 0.06$ & $39.69 \pm 0.15$ \\
\hline 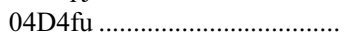 & 0.1330 & $3217.5 \pm 7.0$ & 3297.9 & $3861 \pm 150$ & $21.99 \pm 0.04$ & $0.59 \pm 0.05$ & $38.94 \pm 0.14$ \\
\hline
\end{tabular}

Notes.-All dates are in the observer frame and are expressed as (JD -2,450,000), and the uncertainties in the explosion date are not corrected for time dilation. The magnitudes have been corrected for MW extinction along the line of sight. The large uncertainties for SNLS-03D3ce are due to the fact that it was not observed in $z^{\prime}$, which at its redshift overlaps with rest-frame $I$ band. For this supernova we derived its $V-I$ color by finding the best match in our template day 50 spectra to its observed $g^{\prime} r^{\prime} i^{\prime}$ magnitudes. We then set the dispersion in the $V-I$ color to $50 \%$, which covers the range of all uncorrected colors for the SNe II-P presented in this paper. Our Monte Carlo simulation (see text) was used to derive both the peak values and the uncertainties in the distance moduli. In general, the major contribution to the uncertainty in the distance are (in decreasing significance): explosion date uncertainty, length of extrapolation to day 50, velocity uncertainty, and photometry.

velocities in a manner different from the one presented here (although for high-quality data with strong time constraints, there should be little to no difference between the techniques). In addition, a large fraction of the data in $\mathrm{H} 03$ involves SNe II-P not in the Hubble flow. Our five additional high-redshift supernovae, and one low-redshift SN II-P, increased this pool of data from eight to 14. To address these issues and understand the nature of our data set, its limitations, and the robustness of this result we performed a bootstrap resampling (with replacement) study of the 24 SNe II-P. In Figure 15 we present the results of this study for the variable $\alpha$ in equation (1) and the resulting rms. The rms peaks at 0.26 with a dispersion of 0.05 mag and is well behaved; $\alpha$ peaks at 6.0 with a dispersion about the central peak of 0.5 (similar to that found in the formal $\chi^{2}$ analysis) but shows a long tail to higher values potentially indicating the effect of small number statistics on this analysis.

\section{DISCUSSION}

In this paper we have presented the first Hubble diagram for SNe II-P at cosmologically significant redshifts. The rms scatter of this method, $13 \%$ in distance, compares favorably to the $7 \%-10 \%$ scatter seen in the $\mathrm{SN}$ Ia measurements (Riess et al. 1998; Perlmutter et al. 1999; Astier et al. 2005). This research has reached the stage of the early SNe Ia studies. Further improvements can now be sought with a view to reducing the scatter and increasing the cosmological power of the high-redshift data.

Examples of potential improvements include construction of new light-curve templates to improve estimates of the explosion date. In Figure 10 the color of the SNLS-04D4fu, on initial detection, implies temperatures of $\sim 20,000 \mathrm{~K}$, suggesting this event was observed shortly after explosion and shock breakout, yet at present we do not incorporate this information to help constrain the explosion date. Furthermore, while we observed only five SNe II-P during the first 2 years of the SNLS program, the current photometric data sets contain many well-observed light curves that, once redshifts have been obtained for the host galaxies, will allow us to place tight constraints on how various SNe II-P evolve as a function of time and thus better measure both their plateau colors and the date of explosion.

TABLE 4

Measurements for the Local and Hubble Flow SNe II-P

\begin{tabular}{|c|c|c|c|c|c|}
\hline IAUC Name & $c z$ & $\begin{array}{c}V_{\mathrm{Fe} \mathrm{II}} \\
\left(\mathrm{km} \mathrm{s}^{-1}\right)\end{array}$ & $I$ & $V-I$ & $\mu$ \\
\hline $1986 \mathrm{I} \ldots \ldots$. & 1333 & $3623 \pm 300$ & $13.98 \pm 0.09$ & $0.45 \pm 0.22$ & $30.58 \pm 0.43$ \\
\hline 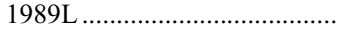 & 1332 & $3529 \pm 300$ & $14.47 \pm 0.05$ & $0.88 \pm 0.07$ & $31.60 \pm 0.38$ \\
\hline $1990 \mathrm{E} \ldots \ldots . .$. & 1426 & $5324 \pm 300$ & $14.51 \pm 0.20$ & $1.31 \pm 0.28$ & $33.24 \pm 0.43$ \\
\hline ................... & 1818 & $6142 \pm 2000$ & $13.87 \pm 0.05$ & $0.58 \pm 0.21$ & $31.96 \pm 0.87$ \\
\hline 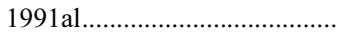 & 4484 & $7330 \pm 2000$ & $16.06 \pm 0.05$ & $0.39 \pm 0.07$ & $34.33 \pm 0.70$ \\
\hline 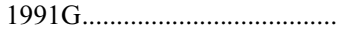 & 1152 & $3347 \pm 500$ & $15.01 \pm 0.09$ & $0.45 \pm 0.11$ & $31.43 \pm 0.53$ \\
\hline 1992af ........................................ & 5438 & $5322 \pm 2000$ & $16.46 \pm 0.20$ & $0.43 \pm 0.28$ & $33.99 \pm 0.99$ \\
\hline 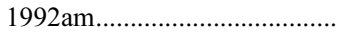 & 14009 & $7868 \pm 300$ & $17.90 \pm 0.05$ & $0.38 \pm 0.07$ & $36.33 \pm 0.12$ \\
\hline 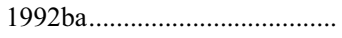 & 1192 & $3523 \pm 300$ & $14.65 \pm 0.05$ & $0.59 \pm 0.07$ & $31.38 \pm 0.41$ \\
\hline 1993A & 8933 & $4290 \pm 300$ & $18.56 \pm 0.05$ & $0.51 \pm 0.07$ & $35.67 \pm 0.20$ \\
\hline 1993S …........... & 9649 & $4569 \pm 300$ & $18.22 \pm 0.05$ & $0.69 \pm 0.07$ & $35.73 \pm 0.18$ \\
\hline 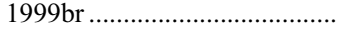 & 848 & $1545 \pm 300$ & $16.67 \pm 0.05$ & $0.84 \pm 0.07$ & $31.70 \pm 0.69$ \\
\hline 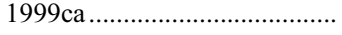 & 3105 & $5353 \pm 2000$ & $15.56 \pm 0.05$ & $0.73 \pm 0.07$ & $33.51 \pm 0.95$ \\
\hline 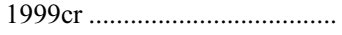 & 6376 & $4389 \pm 300$ & $17.44 \pm 0.05$ & $0.56 \pm 0.07$ & $34.68 \pm 0.20$ \\
\hline 1999 eg & 6494 & $4012 \pm 300$ & $17.72 \pm 0.05$ & $0.55 \pm 0.07$ & $34.71 \pm 0.21$ \\
\hline $1999 \mathrm{em}$ & 917 & $3757 \pm 300$ & $13.28 \pm 0.05$ & $0.57 \pm 0.07$ & $30.15 \pm 0.49$ \\
\hline 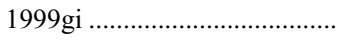 & 901 & $3617 \pm 300$ & $13.95 \pm 0.05$ & $0.91 \pm 0.07$ & $31.18 \pm 0.50$ \\
\hline $2000 \mathrm{cb}$ & 2038 & $4732 \pm 300$ & $15.48 \pm 0.05$ & $0.71 \pm 0.07$ & $33.10 \pm 0.26$ \\
\hline 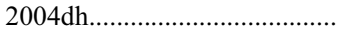 & 5778 & $4990 \pm 300$ & $17.32 \pm 0.05$ & $0.47 \pm 0.07$ & $34.74 \pm 0.18$ \\
\hline
\end{tabular}

Notes.-Data from HP02 and H03, with modifications to SNe 1999em and 1999gi as stated in the text. SN 2004dh is from preliminary work by the CCCP (Gal-Yam et al. 2004, 2006a [in preparation]). All magnitudes have been corrected for MW extinction along the line of sight. 


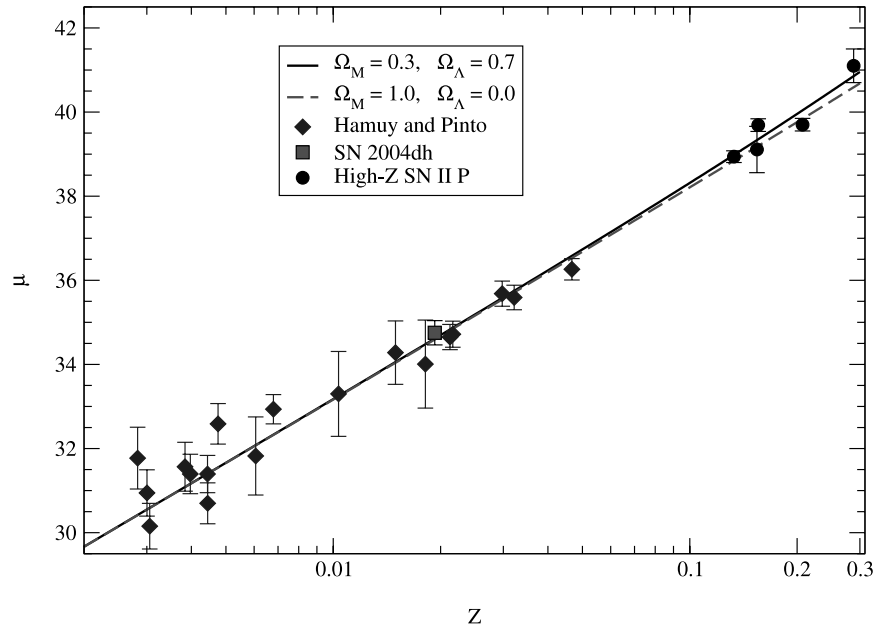

FIG. 13.- Hubble diagram for both the local SNeII-P (diamonds and square) and the high-redshift SNLS SNe II-P (circles) observed spectroscopically with Keck+LRIS. The observed scatter for the $\mathrm{SN}$ in the Hubble flow is $0.26 \mathrm{mag}$ with a reduced $\chi^{2}$ of 1.8 , which indicates an intrinsic dispersion of $0.12 \mathrm{mag}$. To understand the current power of this technique, we have overplotted two differing Hubble lines for a flat cosmology with $\Omega_{M}=1$ and 0.3. [See the electronic edition of the Journal for a color version of this figure.]

In addition, dozens more nearby Hubble flow SNe II-P have been observed by the CCCP and CSP both photometrically and spectroscopically. These data sets will allow us to better understand the velocity evolution of the spectroscopic features on the plateau and see if it is possible to use different photometric bands to standardize the SNe II-P while we improve our current methods. One question that this and future SNe II-P data sets must address is that of Malmquist bias. The two effects this can have on our analysis are a bias on the parameters we have determined in equation (1) and a bias on the discovery of lower luminosity events given the magnitude-limited search. For the latter bias the important parameters to measure are the completeness limit of the search and the intrinsic dispersion on the corrected SNe II-P magnitudes (see Perlmutter et al. 1999 and references therein). From the data presented in this paper (see Fig. 16) it is not clear whether or not Malmquist bias is a major issue or even which

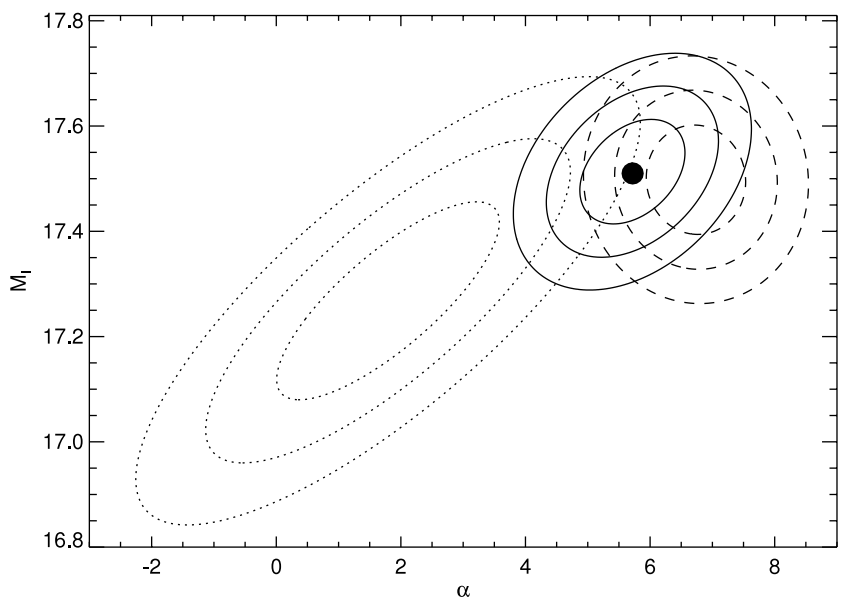

FIG. 14.-Plot of the 1, 2, and $3 \sigma$ confidence contours in the $\alpha-M_{I_{0}}$ plane for the low-redshift only (dashed), high-redshift only (dotted), and combined SNe II-P data sets (solid). Due to low number statistics at both high and low redshift, these parameters are still poorly determined for precision cosmology. Future research by the CCCP and CSP will improve our understanding of this relationship considerably.

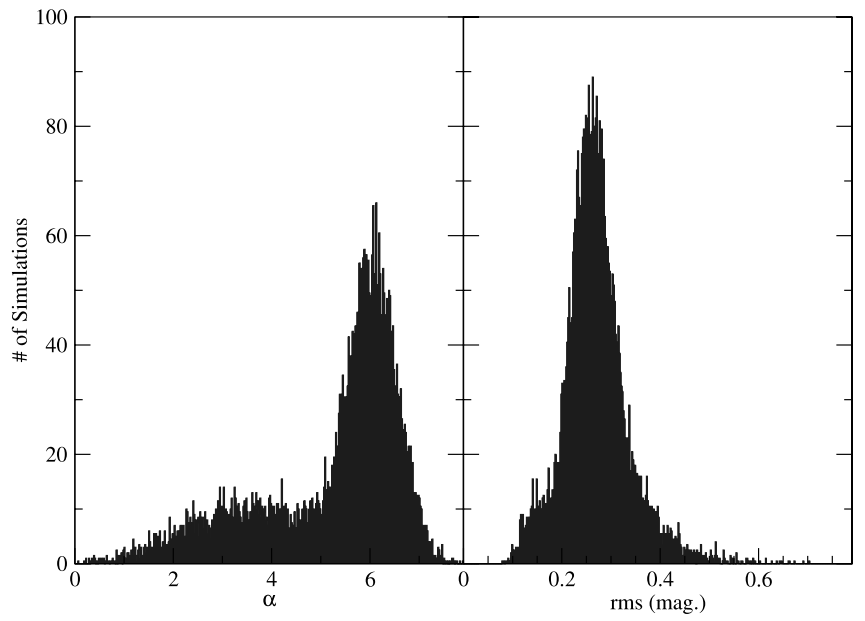

FIG. 15.-Bootstrap resampling (with replacement) study of the variable $\alpha$ and the rms of the 24 SNe II-P presented in this paper. While the rms is nicely behaved, $\alpha$ indicates that our data set may be susceptible to small number statistics. [See the electronic edition of the Journal for a color version of this figure.]

data set, the low-redshift or high-redshift one, suffers from it more. Both the distributions in absolute magnitude and the velocity at day 50 are similar for each set.

Given multicolor light curves $\left(g^{\prime} r^{\prime} i^{\prime} z^{\prime}\right)$ provided by SNLS, we conclude that this method as is can be practically applied, using existing instrumentation, to $z=0.3$. Over this redshift range we could detect the cosmic acceleration at $>95 \%$ level, independent of any other constraints on the cosmological parameters, with $\simeq 12$ additional high-redshift SNe II-P coupled with the five lowredshift CCCP SNe II-P observed this past year. Extending these measurements to $z=0.5$ for the SNLS supernovae would require $J$-band imaging with HST Near-Infrared Camera and MultiObject Spectrometer for average luminosity SNe II-P. However, due to the wide dispersion in their apparent luminosity, a large fraction of this distribution could be observed in the infrared with 8-10 m ground-based telescopes.

Exploring the utility of measuring distances to SNe II-P has potential benefits well beyond simply verifying, independently, the acceleration seen at redshifts $z<1$. Several plausible models for the time evolution of the dark energy require distance measures to $z \simeq 2$ and beyond (Linder \& Huterer 2003). At such

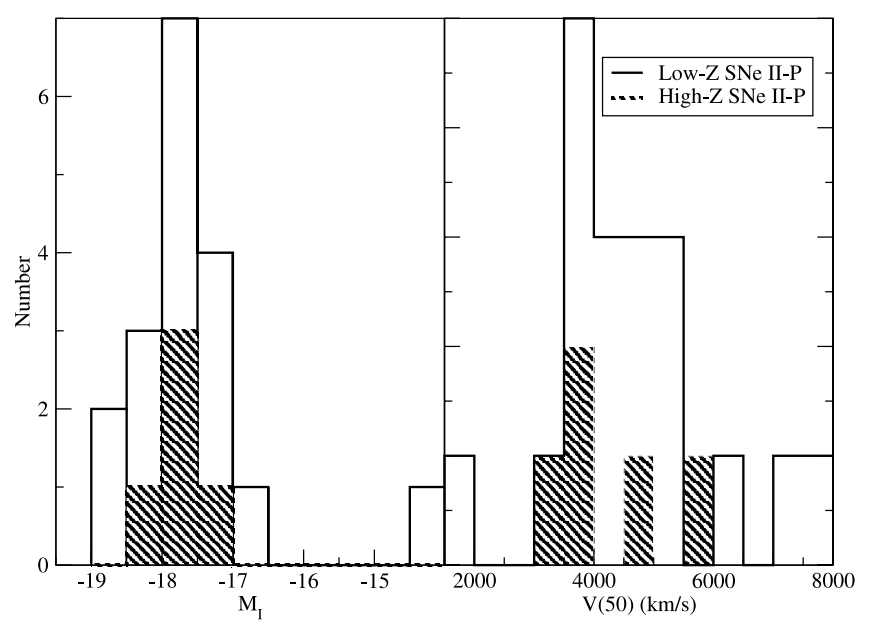

FIG. 16.- Histograms of the observed absolute magnitude in $I$ band, uncorrected for extinction, and the day 50 velocities, for both the high-redshift ( filled) and low-redshift (open) SNe II-P. Future data sets will have to address the important issue of Malmquist bias, a question beyond the scope of this paper. 
high redshifts, other cosmological probes may become less effective than at $z \leq 1$. Weak lensing, for example, will suffer from the loss of suitable lenses, and while evidence at $z<1$ suggests that some fraction of SNe Ia explode with very short delay times and hence will be abundant at high-redshift (Mannucci et al. 2005; M. Sullivan et al. 2006, in preparation), the efficiency and metallicity dependence of the SN Ia progenitor system is still in doubt and may curtail their production (e.g., Kobayashi et al. 1998). However, current models for the cosmic star formation history predict an abundant source of SNe II at these epochs, and future facilities, such as the proposed Joint Dark Energy Mission telescope, in concert with James Webb Space Telescope and/or future $30 \mathrm{~m}$ telescopes such as the Thirty Meter Telescope, could potentially use SNe II-P to determine distances at these very high redshifts.

Based on observations obtained with MegaPrime/MegaCam, a joint project of CFHT and CEA/DAPNIA, at the CanadaFrance-Hawaii Telescope (CFHT), which is operated by the National Research Council (NRC) of Canada, the Institut National des Science de 1'Univers of the Centre National de la Recherche Scientifique (CNRS) of France, and the University of Hawaii. This work is based in part on data products produced at the Canadian Astronomy Data Centre as part of the CFHT Legacy Survey, a collaborative project of the NRC and CNRS. Some of the data presented herein were obtained at the W. M. Keck Observatory, which is operated as a scientific partnership among the California Institute of Technology, the University of California, and the National Aeronautics and Space Administration. The Observatory was made possible by the generous financial support of the W. M. Keck Foundation. The authors wish to recognize and acknowledge the very significant cultural role and reverence that the summit of Mauna Kea has always had within the indigenous Hawaiian community. We are most fortunate to have the opportunity to conduct observations from this mountain. P. E. N. acknowledges support from NASA ATP and LTSA grants. R. S. E. acknowledges financial support from the Department of Energy (DOE). A. G. acknowledges support by NASA through Hubble Fellowship grant HST-HF-01158.01-A, awarded by STScI, which is operated by AURA, Inc., for NASA, under contract NAS5-26555. D. C. L. is supported by a National Science Foundation (NSF) Astronomy and Astrophysics Postdoctoral Fellowship under award AST 04-01479. This research used resources of the National Energy Research Scientific Computing Center, which is supported by the Office of Science of the US DOE, under contract DE-AC03-76SF00098. We thank them for a generous allocation of computing time.
Astier, P., et al. 2006, A\&A, 447, 31

Baron, E., Nugent, P. E., Branch, D., \& Hauschildt, P. H. 2004, ApJ, 616, L91 Baron, E., Nugent, P., Branch, D., Hauschildt, P. H., Leonard, D., Filippenko, A. V., Turatto, M., \& Cappellaro, E. 2003, ApJ, 586, 1199

Benetti, S. 1991, in Supernova 1987A and Other Supernovae, ed. I. J. Danziger \& K. Kjaaumlr (Garching: ESO), 339

Bennett, C. L., et al. 2003, ApJS, 148, 1

Cappellaro, E., et al. 2005, A\&A, 430, 83

Chugai, N. N. 1994, in Circumstellar Media in Late Stages of Stellar Evolution, ed. R. E. S. Clegg, I. R. Stevens, \& W. P. S. Meikle (Cambridge: Cambridge Univ. Press), 148

Efstathiou, G., et al. 2002, MNRAS, 330, L29

Eisenstein, D. J., Blanton, M., Zehavi, I., Bahcall, N., Brinkmann, J., Loveday, J., Meiksin, A., \& Schneider, D. 2005, ApJ, 619, 178

Freedman, W. L. 2005, in ASP Conf. Ser. 339, Observing Dark Energy, ed. S. C. Wolff \& T. R. Lauer (San Francisco: ASP), 50

Gal-Yam, A., Cenko, S. B., Fox, D. W., Leonard, D. C., Moon, D.-S., Sand, D. J., \& Soderberg, A. M. 2004, AAS Meeting, 205, 40.06

Guy, J., Astier, P., Nobili, S., Regnault, N., \& Pain, R. 2005, A\&A, 443, 781

Hamuy, M. 2002, Ph.D. thesis, Univ. Arizona

2003, in IAU Colloq. 192, Cosmic Explosions: On the 10th Anniversary of SN1993J, ed. J.-M. Marcaide \& K. W. Weiler (Berlin: Springer), $535(\mathrm{H} 03)$

Hamuy, M., \& Pinto, P. A. 2002, ApJ, 566, L63 (HP02)

Hamuy, M., et al. 2001, ApJ, 558, 615

Heger, A., Fryer, C. L., Woosley, S. E., Langer, N., \& Hartmann, D. H. 2003, ApJ, 591, 288

Kirshner, R. P., \& Kwan, J. 1974, ApJ, 193, 27

Knop, R. A., et al. 2003, ApJ, 598, 102

\section{EFERENCES}

Kobayashi, C., Tsujimoto, T., Nomoto, K., Hachisu, I., \& Kato, M. 1998, ApJ, 503, L155

Leonard, D. C. 2000, Ph.D. thesis, Univ. California, Berkeley

Leonard, D. C., \& Filippenko, A. V. 2005, in ASP Conf. Ser. 342, 1604-2004: Supernovae as Cosmological Lighthouses, ed. S. B. M. Turatto et al. (San Francsico: ASP), 330

Leonard, D. C., Kanbur, S. M., Ngeow, C. C., \& Tanvir, N. R. 2003, ApJ, 594, 247

Leonard, D. C., et al. 2002, AJ, 124, 2490

Li, W., Van Dyk, S. D., Filippenko, A. V., \& Cuillandre, J.-C. 2005, PASP, 117, 121

Linder, E. V., \& Huterer, D. 2003, Phys. Rev. D, 67, 081303

Mannucci, F., della Valle, M., Panagia, N., Cappellaro, E., Cresci, G., Maiolino, R., Petrosian, A., \& Turatto, M. 2005, A\&A, 433, 807

Mitchell, R., et al. 2002, ApJ, 574, 293

Moore, M., \& Li, W. 2004, IAU Circ., 8375, 2

Nugent, P., Kim, A., \& Perlmutter, S. 2002, PASP, 114, 803

Oke, J. B., et al. 1995, PASP, 107, 375

Perlmutter, S., et al. 1999, ApJ, 517, 565

Riess, A., et al. 1998, AJ, 116, 1009

Schmidt, B. P. 1993, Ph.D. thesis, Harvard Univ.

Smartt, S. J., Maund, J. R., Gilmore, G. F., Tout, C. A., Kilkenny, D., \& Benetti, S. 2003, MNRAS, 343, 735

Sullivan, M., et al. 2005, in ASP Conf. Ser. 342, 1604-2004: Supernovae as Cosmological Lighthouses, ed. S. B. M. Turatto et al. (San Francisco: ASP), 466 Tonry, J. L., Blakeslee, J. P., Ajhar, E. A., \& Dressler, A. 2000, ApJ, 530, 625 Tonry, J. L., \& Davis, M. 1979, AJ, 84, 1511

Turatto, M., Cappellaro, E., Benetti, S., \& Danziger, I. J. 1993, MNRAS, 265, 471 